\title{
Crystal structure of $N, N^{\prime}$-bis(2-methyl-4-phenyl-5-tert-butyl- imidazo[1,5-b]pyridazin-7-yl)ethylenediamine, $\mathrm{C}_{2} \mathrm{H}_{4}(\mathrm{NH})_{2}\left(\mathrm{C}_{17} \mathrm{H}_{18} \mathrm{~N}_{3}\right)_{2}$
}

\author{
T. Irrgang, S. Balireddi, S. Karthikeyan and R. Kempe* \\ Universität Bayreuth, Lehrstuhl für Anorganische Chemie II, 95440 Bayreuth, Germany
}

Received November 28, 2005, accepted and available on-line December 14, 2005; CCDC no. 1267/1689

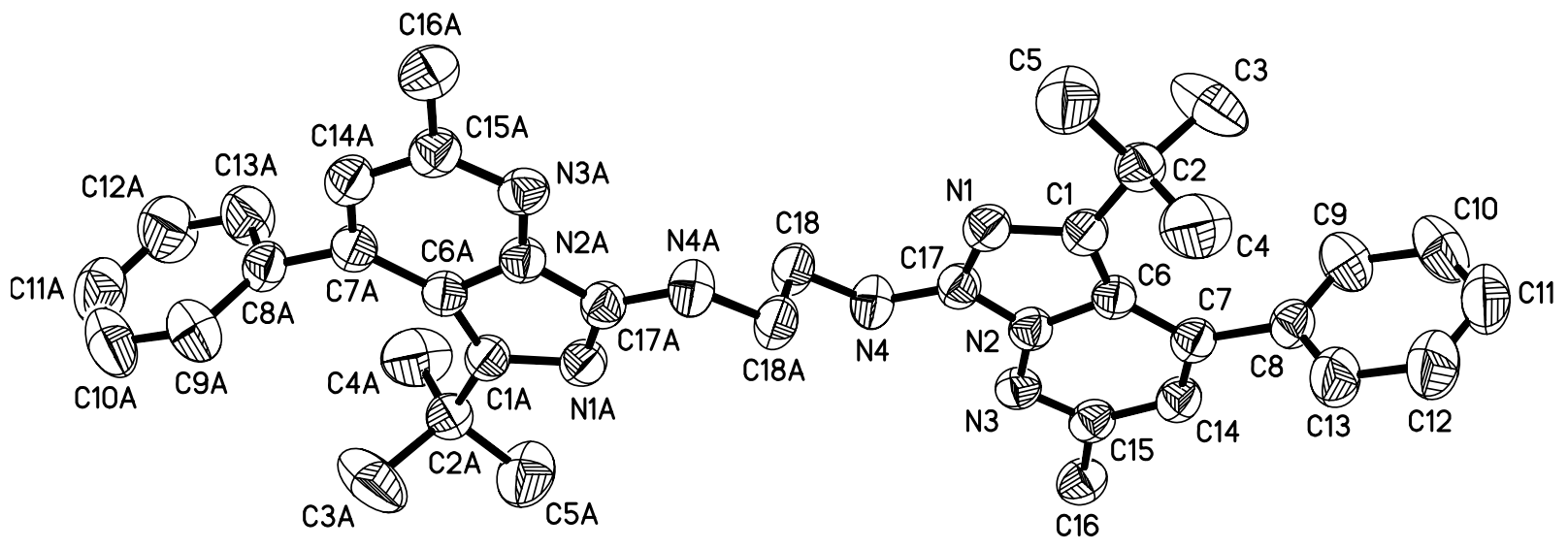

\begin{abstract}
$\mathrm{C}_{36} \mathrm{H}_{42} \mathrm{~N}_{8}$, monoclinic, $P 121 / c 1$ (no. 14), $a=14.004(3) \AA$, $b=9.164(2) \AA, c=13.468(3) \AA, \beta=109.30(3)^{\circ}$,

$V=1631.3 \AA^{3}, Z=2, R_{\mathrm{gt}}(F)=0.060, w R_{\mathrm{ref}}\left(F^{2}\right)=0.158$, $T=193 \mathrm{~K}$.
\end{abstract}

\section{Source of material}

The title compound was synthesized in accordance to a published procedure [1]. Re-crystallization from ethanol/chloroform ( $v / v$ 2:1) gave orange crystals suitable for X-ray structure analysis.

\section{Experimental details}

The H4N atom was included in the refinement process due to the importance with regard to the hydrogen bond pattern.

\section{Discussion}

We introduced a series of new imidazo[1,5- $b]$ pyridazine substituted diamines [1-3] (for instance the title compound) as novel amido ligands. The deprotonated diamines can act as bisamido ligands and bind early and late transition metals as a five-membered chelate [1]. The significant shorter N3-C15 (1.307 $\AA$ ) and C7-C14 (1.361 $\AA$ ) bonds indicate the localization of the double bonds of the six-membered pyridazine ring. The deviation from plane of the imidazopyridazine is $0.011 \AA$. The 7-substituted amido N4 atom is in the plane with the imidazopyridazine (only $0.001 \AA$ deviation above). The phenyl substituent in the 4-position is twisted with regard to the imidazo[1,5-b]pyridazine with dihedral angle of $94.7^{\circ}$, respectively. The title compound is predicted to form inter- or intramolecular $\mathrm{H}$ bonds, because it contains a potential proton donor and acceptor functionalities. The amino $\mathrm{H}$ atoms form intramolecular hydrogen bond to the corresponding pyridazine $\mathrm{N} 3$ atoms $(d(\mathrm{H} 4 \mathrm{~N} \cdots \mathrm{N} 3)=2.442 \AA)$.
Table 1. Data collection and handling.

$$
\begin{array}{ll}
\text { Crystal: } & \text { orange prism, size } 0.3 \times 0.4 \times 0.5 \mathrm{~mm} \\
\text { Wavelength: } & \text { Mo } K_{\alpha} \text { radiation }(0.71073 \AA) \\
\mu: & 0.73 \mathrm{~cm}^{-1} \\
\text { Diffractometer, scan mode: } & \text { Stoe IPDS II, } \omega \\
2 \theta_{\text {max }}: & 51.66^{\circ} \\
N(h k l)_{\text {measured }}, N(h k l)_{\text {unique }}: & 20975,3103 \\
\text { Criterion for } I_{\text {obs }}, N(h k l)_{\mathrm{gt}}: & I_{\text {obs }}>2 \sigma\left(I_{\mathrm{obs}}\right), 2204 \\
N(\text { param })_{\text {refined }}: & 206 \\
\text { Programs: } & \text { SIR97 [4], SHELXL-97 [5] }
\end{array}
$$

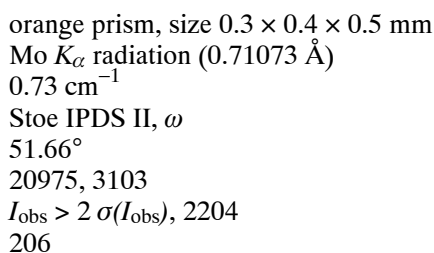

Table 2. Atomic coordinates and displacement parameters (in $\AA^{2}$ ).

\begin{tabular}{llllll}
\hline Atom & Site & $x$ & $y$ & $z$ & $U_{\text {iso }}$ \\
\hline & & & & & \\
$\mathrm{H}(3 \mathrm{~A})$ & $4 e$ & 0.6491 & -0.0598 & 0.3075 & 0.149 \\
$\mathrm{H}(3 \mathrm{~B})$ & $4 e$ & 0.5603 & 0.0311 & 0.3290 & 0.149 \\
$\mathrm{H}(3 \mathrm{C})$ & $4 e$ & 0.6640 & 0.0023 & 0.4227 & 0.149 \\
$\mathrm{H}(4 \mathrm{~A})$ & $4 e$ & 0.6444 & 0.1148 & 0.1620 & 0.137 \\
$\mathrm{H}(4 \mathrm{~B})$ & $4 e$ & 0.6590 & 0.2857 & 0.1869 & 0.137 \\
$\mathrm{H}(4 \mathrm{C})$ & $4 e$ & 0.5568 & 0.2063 & 0.1854 & 0.137 \\
$\mathrm{H}(5 \mathrm{~A})$ & $4 e$ & 0.5744 & 0.2941 & 0.3574 & 0.130 \\
$\mathrm{H}(5 \mathrm{~B})$ & $4 e$ & 0.6765 & 0.3778 & 0.3635 & 0.130 \\
$\mathrm{H}(5 \mathrm{C})$ & $4 e$ & 0.6754 & 0.2672 & 0.4550 & 0.130 \\
$\mathrm{H}(9)$ & $4 e$ & 0.7558 & -0.3006 & 0.2930 & 0.079 \\
$\mathrm{H}(10)$ & $4 e$ & 0.6160 & -0.4183 & 0.1765 & 0.097 \\
$\mathrm{H}(11)$ & $4 e$ & 0.5433 & -0.3320 & 0.0065 & 0.100 \\
$\mathrm{H}(12)$ & $4 e$ & 0.6126 & -0.1312 & -0.0491 & 0.112 \\
$\mathrm{H}(13)$ & $4 e$ & 0.7526 & -0.0145 & 0.0659 & 0.088 \\
$\mathrm{H}(14)$ & $4 e$ & 0.9523 & -0.2093 & 0.2288 & 0.059 \\
$\mathrm{H}(16 \mathrm{~A})$ & $4 e$ & 1.1975 & -0.0695 & 0.3845 & 0.086 \\
$\mathrm{H}(16 \mathrm{~B})$ & $4 e$ & 1.1478 & -0.1263 & 0.2662 & 0.086 \\
$\mathrm{H}(16 \mathrm{C})$ & $4 e$ & 1.1466 & -0.2278 & 0.3625 & 0.086 \\
$\mathrm{H}(18 \mathrm{~A})$ & $4 e$ & 1.0774 & 0.4685 & 0.5977 & 0.063 \\
$\mathrm{H}(18 \mathrm{~B})$ & $4 e$ & 0.9598 & 0.4355 & 0.5746 & 0.063 \\
$\mathrm{H}(4 \mathrm{~N})$ & $4 e$ & $1.085(2)$ & $0.274(3)$ & $0.498(2)$ & $0.057(8)$ \\
& & & & &
\end{tabular}

\footnotetext{
* Correspondence author (e-mail: kempe@uni-bayreuth.de)
} 
Table 3. Atomic coordinates and displacement parameters (in $\AA^{2}$ ).

\begin{tabular}{|c|c|c|c|c|c|c|c|c|c|c|}
\hline Atom & Site & $x$ & $y$ & $z$ & $U_{11}$ & $U_{22}$ & $U_{33}$ & $U_{12}$ & $U_{13}$ & $U_{23}$ \\
\hline $\mathrm{C}(1)$ & $4 e$ & $0.7917(2)$ & $0.1519(2)$ & $0.3522(2)$ & $0.054(1)$ & $0.041(1)$ & $0.042(1)$ & $-0.001(1)$ & $0.017(1)$ & $0.001(1)$ \\
\hline$C(2)$ & $4 e$ & $0.6771(2)$ & $0.1615(3)$ & $0.3199(2)$ & $0.052(1)$ & $0.049(1)$ & $0.055(1)$ & $-0.001(1)$ & $0.021(1)$ & $-0.003(1)$ \\
\hline$C(3)$ & $4 e$ & $0.6338(2)$ & $0.0214(4)$ & $0.3472(4)$ & $0.070(2)$ & $0.073(2)$ & $0.175(4)$ & $-0.004(2)$ & $0.067(2)$ & $0.018(2)$ \\
\hline$C(4)$ & $4 e$ & $0.6302(2)$ & $0.1951(5)$ & $0.2032(2)$ & $0.055(2)$ & $0.143(3)$ & $0.069(2)$ & $0.005(2)$ & $0.011(1)$ & $0.003(2)$ \\
\hline$C(5)$ & $4 e$ & $0.6483(2)$ & $0.2863(4)$ & $0.3792(3)$ & $0.061(2)$ & $0.092(2)$ & $0.108(3)$ & $0.012(2)$ & $0.027(2)$ & $-0.028(2)$ \\
\hline$C(6)$ & $4 e$ & $0.8565(2)$ & $0.0546(2)$ & $0.3266(2)$ & $0.050(1)$ & $0.041(1)$ & $0.038(1)$ & $-0.003(1)$ & $0.0149(9)$ & $0.002(1)$ \\
\hline$C(7)$ & $4 e$ & $0.8585(2)$ & $-0.0703(2)$ & $0.2640(2)$ & $0.056(1)$ & $0.040(1)$ & $0.040(1)$ & $-0.001(1)$ & $0.017(1)$ & $0.003(1)$ \\
\hline $\mathrm{C}(8)$ & $4 e$ & $0.7668(2)$ & $-0.1431(3)$ & $0.1920(2)$ & $0.054(1)$ & $0.042(1)$ & $0.054(1)$ & $-0.001(1)$ & $0.019(1)$ & $-0.009(1)$ \\
\hline $\mathrm{C}(9)$ & $4 e$ & $0.7265(2)$ & $-0.2646(3)$ & $0.2233(2)$ & $0.059(2)$ & $0.055(2)$ & $0.080(2)$ & $-0.004(1)$ & $0.016(1)$ & $0.008(1)$ \\
\hline $\mathrm{C}(10)$ & $4 e$ & $0.6435(2)$ & $-0.3348(3)$ & $0.1540(3)$ & $0.060(2)$ & $0.054(2)$ & $0.125(3)$ & $-0.013(1)$ & $0.027(2)$ & $-0.007(2)$ \\
\hline $\mathrm{C}(11)$ & $4 e$ & $0.6009(2)$ & $-0.2846(4)$ & $0.0535(3)$ & $0.064(2)$ & $0.087(2)$ & $0.091(2)$ & $-0.012(2)$ & $0.013(2)$ & $-0.034(2)$ \\
\hline $\mathrm{C}(12)$ & $4 e$ & $0.6415(3)$ & $-0.1658(4)$ & $0.0209(3)$ & $0.089(2)$ & $0.114(3)$ & $0.062(2)$ & $-0.029(2)$ & $0.006(2)$ & $-0.012(2)$ \\
\hline $\mathrm{C}(13)$ & $4 e$ & $0.7243(2)$ & $-0.0962(4)$ & $0.0896(2)$ & $0.080(2)$ & $0.081(2)$ & $0.052(2)$ & $-0.024(2)$ & $0.012(1)$ & $-0.002(1)$ \\
\hline $\mathrm{C}(14)$ & $4 e$ & $0.9504(2)$ & $-0.1253(3)$ & $0.2694(2)$ & $0.057(2)$ & $0.045(1)$ & $0.047(1)$ & $0.001(1)$ & $0.018(1)$ & $-0.003(1)$ \\
\hline $\mathrm{C}(17)$ & $4 e$ & $0.9438(2)$ & $0.2270(2)$ & $0.4374(2)$ & $0.051(1)$ & $0.041(1)$ & $0.042(1)$ & $-0.002(1)$ & $0.015(1)$ & $0.000(1)$ \\
\hline $\mathrm{C}(18)$ & $4 e$ & $1.0136(2)$ & $0.4411(3)$ & $0.5421(2)$ & $0.065(2)$ & $0.046(1)$ & $0.045(1)$ & $-0.005(1)$ & $0.014(1)$ & $-0.009(1)$ \\
\hline $\mathrm{N}(1)$ & $4 e$ & $0.8477(2)$ & $0.2573(2)$ & $0.4198(2)$ & $0.056(1)$ & $0.045(1)$ & $0.047(1)$ & $0.0008(9)$ & $0.0198(9)$ & $-0.0012(9)$ \\
\hline $\mathrm{N}(2)$ & $4 e$ & $0.9544(1)$ & $0.1072(2)$ & $0.3838(1)$ & $0.047(1)$ & $0.042(1)$ & $0.041(1)$ & $-0.0007(8)$ & $0.0151(8)$ & $0.0006(8)$ \\
\hline $\mathrm{N}(3)$ & $4 e$ & $1.0476(1)$ & $0.0533(2)$ & $0.3902(1)$ & $0.049(1)$ & $0.046(1)$ & $0.043(1)$ & $0.0019(9)$ & $0.0177(8)$ & $0.0031(9)$ \\
\hline $\mathrm{N}(4)$ & $4 e$ & $1.0259(2)$ & $0.2990(2)$ & $0.5012(2)$ & $0.054(1)$ & $0.048(1)$ & $0.056(1)$ & $-0.001(1)$ & $0.012(1)$ & $-0.009(1)$ \\
\hline
\end{tabular}

Acknowledgment. We thank the Fonds der Chemischen Industrie for financial support.

\section{References}

1. Irrgang, T.; Kempe, R.: Early and Late Transition Metal Complexes Stabilised by Imidazopyridazine-Substituted Bisamido Ligands. Eur. J. Inorg. Chem. (2005) 4382-4392.

2. Irrgang, T.; Kempe, R.: Crystal structure of $N, N^{\prime}$-bis(2,4-dimethyl-5phenylimidazo[1,5-b]pyridazin-7-yl)-1,3-diaminopropane, $\mathrm{C}_{31} \mathrm{H}_{32} \mathrm{~N}_{8}$. Z . Kristallogr. NCS 220 (2005) 403-404.

3. Irrgang, T.; Karthikeyan, S.; Balireddi, S.; Kempe, R.: Crystal structure of $N, N^{\prime}$-bis(2-methyl-4,5-diphenylimidazo[1,5-b]pyridazin-7-yl)-1,3-di- aminopropane ethanol solvate, $\mathrm{C}_{3} \mathrm{H}_{6}(\mathrm{NH})_{2}\left(\mathrm{C}_{19} \mathrm{H}_{14} \mathrm{~N}_{3}\right)_{2} \cdot \mathrm{C}_{2} \mathrm{H}_{5} \mathrm{OH}$. Z. Kristallogr. NCS 221 (2006) 20-22.

4. Altomare, A.; Burla, M. C.; Camalli, M.; Cascarano, G. L.; Giacovazzo, C.; Guagliardi, A.; Moliterni, A. G. G.; Polidori, G.; Spagna, R.: SIR97: a new tool for crystal structure determination and refinement. J. Appl. Crystallogr. 32 (1999) 115-119.

5. Sheldrick, G. M.: SHELXL-97. Program for the Refinement of Crystal Structures. University of Göttingen, Germany 1997. 